\title{
The Lung and Soft Tissue Stopping Power Estimates for a Modified Phantom Using MCNPX
}

\author{
Olaseni M. Bello ${ }^{1,2}$ \\ ${ }^{1}$ Physics Department, Universiti Technologi Malaysia, UTM, Johor Bahru, Malaysia \\ ${ }^{2}$ Physics Department, Nigeria Police Academy, Nigeria \\ Wan Mohammad S. Wan Hassan ${ }^{1} \&$ Norehan M. Nor ${ }^{1}$ \\ ${ }^{1}$ Physics Department, Universiti Technologi Malaysia, UTM, Malaysia
}

\begin{abstract}
The Adult Male ${ }^{\circledR}$ and Adult Female ${ }^{\circledR}$ phantoms of ORNL were modified with Ir-192 source and additional tally cards from the MCNP® data library. The lung and eleven soft tissues were used for the study. The soft tissues include Liver, Stomach, Ovaries, Testes, Brain, Thyroid, Kidney, Pancreas, Gall Bladder, Heart, and the Small Intestine. The computational simulation was achieved using MCNPX with nps of $10^{7}$, and the data set of the lung and soft tissues derived for Malaysian population [14]. The relative error, $R$ was $<0.05$ for $F 4$ and the $F 6$ tallies across the energy range studied. The results obtained for 0.1-10.0 MeV energies for the F4 and F6 tallies were used to estimate the mass stopping power, $\mathrm{S} / \mathrm{\rho}(\mathrm{MeVg}$ $\left.{ }^{1} \mathrm{~cm}^{2}\right)$ and the linear stopping power, $\mathrm{S}(\mathrm{MeV} / \mathrm{cm})$. The result compares favorably among the tissues. This result is very relevant and useful in validating an on-going lung and soft tissue model construction.
\end{abstract}

Keywords:- Stopping Power, Radiation Phantom, MCNPX, Tissues, Photon Depth, Photon Area Density

\section{INTRODUCTION}

When a medium is exposed to ionizing radiation and photon energy passes through it, the inelastic collisions that occur greatly characterize the particle transport [1]. The energy loss during the transport process [2] account for the continuous slowing down approximation, CSDA called stopping power [1,2]. The stopping power is rarely measured [3] but it has been measured for protons [4-8], electron interactions with humans [9] and from first principle to enhance research validation and empiricism reduction [10]

\section{A. Linear Stopping Power, $S$}

The linear stopping power, $S$ is the energy loss per unit length, $\frac{d E}{d x}$ [3] or average energy lost by charged particles per unit path length, $-\frac{d E}{d x}[1]$. Unit: $\mathrm{MeV} / \mathrm{cm}$.

\section{B. Mass Stopping Power, $S / \rho$}

$\frac{1}{\rho} \mathrm{S}_{\mathrm{el}}=-\left(\frac{\mathrm{dE}}{\rho \mathrm{ds}}\right)_{\mathrm{el}}=\mathrm{N}_{\mathrm{a}} \mathrm{Z} \int_{\mathrm{W}_{\min }}^{\mathrm{W}_{\max }} \mathrm{W} \frac{\mathrm{d} \sigma}{\mathrm{dW}} \mathrm{dW}$

and $\mathrm{S} / \rho$ is $\frac{1}{\rho} \frac{d E}{d x}[2]$. Unit: $\mathrm{MeVg}^{-1} \mathrm{~cm}^{2}$.

Rearranging the unit, $\quad \frac{\mathrm{s}}{\rho}=\frac{\mathrm{MeV}}{\left(\mathrm{g} / \mathrm{cm}^{2}\right)}$

\section{METHOD AND MATERIAL}

Ir-192[11] and additional tallies were used for the modification, MCNPX F4 measure the fluence, $\mathrm{cm}^{-2}$ and F6 measure $\mathrm{MeV} / \mathrm{g}[12,13]$. The tissue mass and density are presented in Table 1, the table is the derived data set for Malaysia population [14].

Dimensionally, $\frac{\text { Tally } 6}{\text { Tally } 4}=\frac{\mathrm{s}}{\rho}\left(\mathrm{MeVg}^{-1} \mathrm{~cm}^{2}\right)$

The source is $1 \mathrm{~m}$ AP from the trunk of the phantom. The AP geometry specify that the photons are incident on the front of the phantom [15].

\begin{tabular}{|c|c|c|c|}
\hline Tissue & Male, $\mathrm{g}$ & Female, $\mathrm{g}$ & Density, $\rho$ \\
\hline Lung & 1110.26 & 711.00 & 0.25 \\
\hline Liver & 1729.31 & 539.35 \\
\hline Stomach Wall & 147.70 & 110.68 \\
\hline Testes & 37.00 & - \\
\hline Ovaries & - & 10.32 \\
\hline Brain & 1470.25 & 1179.10 \\
\hline Thyroid & 19.85 & 16.88 \\
\hline Kidney & 322.98 & 254.20 \\
\hline Pancreas & 119.10 & 79.73 \\
\hline Spleen & 161.49 & 144.45 \\
\hline Gall Bladder & 9.08 & 7.50 \\
\hline Heart & 147.70 & 110.68 \\
\hline
\end{tabular}

Table 1:- Table of lung and soft tissues mass and density [14] 


\section{A. The Calculation}

The values of the F4 and the F6 output results are obtained directly from the MCNPX result after simulation. The relative error, $\mathrm{R}$ for the $\mathrm{F} 4$ and $\mathrm{F} 6$ data retrieved from the tally fluctuation chart is $<0.05$ for the photon energies studied. The ratio of F6 to F4 yield the mass stopping power while the product of the mass stopping power and the density yield the linear stopping power. The Figure 1 show the flow of the calculation.

\section{B. The Design Flow Chart}

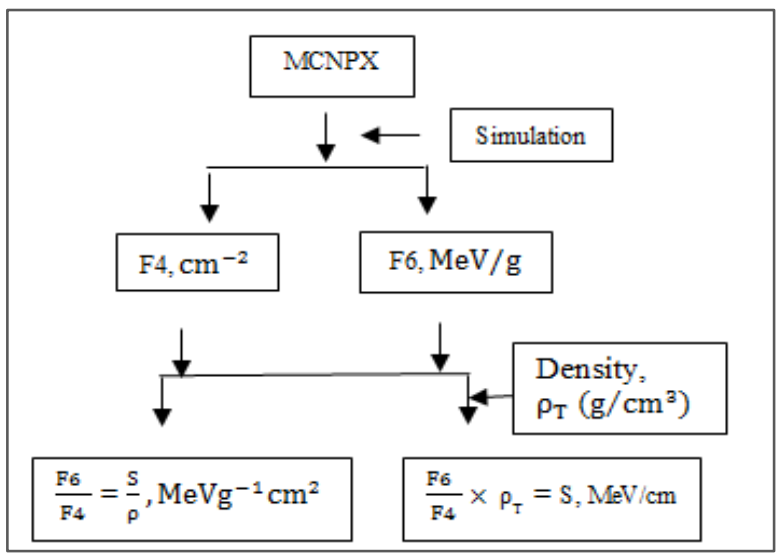

Fig 1:- Schematic Design of the Study Estimates

\section{THE RESULT AND DISCUSSION}

The linear stopping power and the mass stopping power recorded for the modified female phantom is greater than that of the male phantom by about $0.038 \%$ on the average at lower energies $\leq 0.1 \mathrm{MeV}$ while it is on average of $0.1 \%$ less at higher energies $\geq 0.2 \mathrm{MeV}$. The linear stopping power is an estimate of the energy and the depth the photon travels in the tissue while the mass stopping power is a pointer to the energy and the area covered by the photon when traversing a tissue. Despite lower mass of the female tissues, compare to the male; it is evident that the photons travel deeper and cover wider area at lower energies $\leq 0.1 \mathrm{MeV}$ in the female than in the male. However, the pattern is reversed at higher energies. This observed pattern is consistent for all the tissues studied. The Table 2 to Table 5 present the values of $S$ and $S / \rho$ for the male and the female.

The curves of the mass stopping power is presented in figure 2. The $\mathrm{S} / \rho$ vs $\mathrm{MeV}$ curve flattens after a certain energy point. This flattening behavior is also reported in [16] as it is considered a very important characteristic. The point is defined in [3] as $\mathrm{E}_{\mathrm{k}} \approx 3 \mathrm{~m}_{\mathrm{e}} \mathrm{c}^{2}$.

\section{CONCLUSION}

The estimation of the linear stopping power and the mass stopping power is a very important tool in validating the result from experiment. This result, obtained from MCNPX idealized photon exposure is very crucial and relevant as it will serve as a basis for justifying the result from experiment.

\section{RECOMMENDATION}

The authors recommend further efforts in estimating the linear and the mass stopping powers at energies $>10 \mathrm{MeV}$ but within the medical diagnostic energy range.

\section{ACKNOWLEDGMENT}

This work was supported by:

Tertiary Education Trust Fund (TETFund), Nigeria and

TWAS-COMSTECH Joint Research Grant, Reference: 18-326 RG/ITC/AS_C-FR3240305782

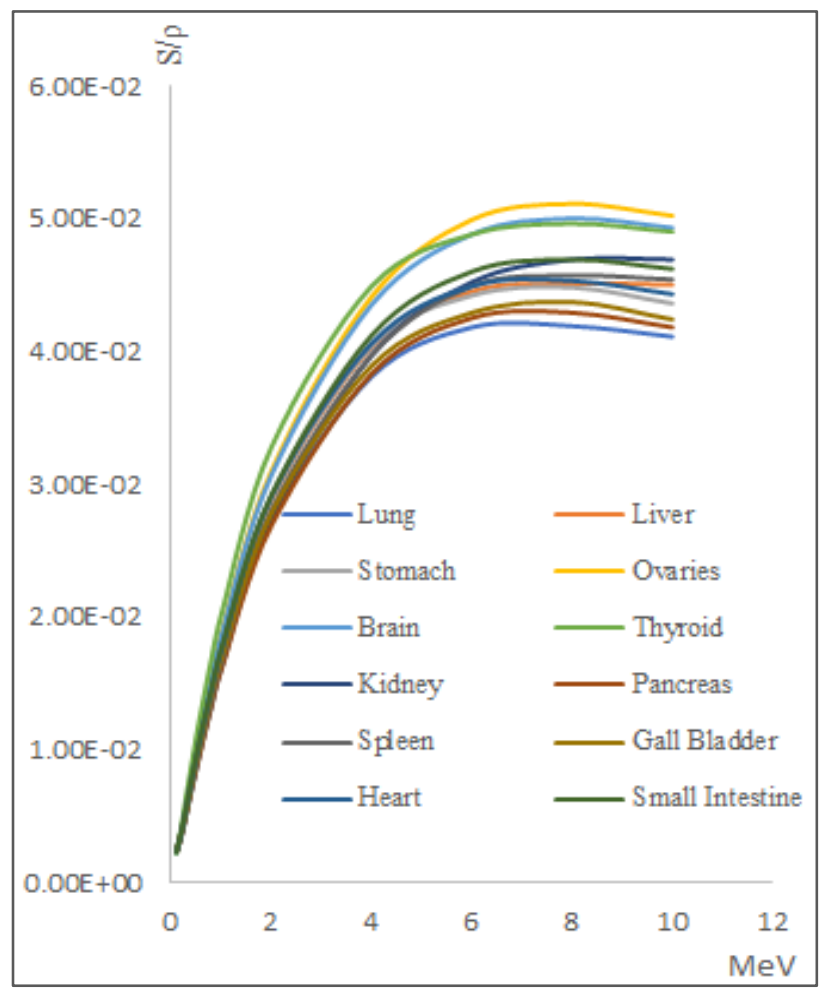

Fig 2:- $S / \rho$ vs Energy

\section{REFERENCES}

[1]. Oleg N.Vassiliev, 2017. Monte Carlo Methods for Radiation Transport: Fundamentals and Advanced Topics. Springer, Switzerland.

[2]. Pedro A., David T. B., Alan E. N., Jan S., and Frank A. H., 2017. Fundamentals of Ionizing Radiation Dosimetry. Willey-VCH Verlag GmbH \& Co., Printed in the UK.

[3]. Seuntjens J. P., Strydom W. and Shortt K. R., 2003 Chapter 2: Dosimetric Principles, Quantities and Units, in: Ervin B. P. (Ed) Review of Radiation Oncology Physics: A Handbook for Teachers and Students. IAEA, Austria. Pp 38 - 52

[4]. Damache S., Djaroum S., Ouichaoui S., Amari L and Moussa D. (2017) Stopping power and energy loss straggling for $0.9-3.4 \mathrm{MeV}$ protons in a Kapton polyimide thin film. https://arxiv.org/ftp/arxiv/papers/1709/1709.06083.pdf (accessed 27 June, 2020) 
[5]. Anthony K. S. A., Godsway B. K., Nayaaba R. A., Eric M. N., 2017. A Theoretical Study of Stopping Power and Range for Low Energy <3.0MeV Protons in Aluminium, Germanium, Lead, Gold and Copper Solid Materials. Open Science Journal 2(2).

[6]. Ahmet Bozkurt, 2017. Monte Carlo calculation of proton stopping power and ranges in water for therapeutic energies. EPJ Web of Conferences 154, 01007. http://DOI:0.1051/epjconf/201715401007. TESNAT 2017

[7]. Gooley T., Richard E. P. and Highes G. H., 2003. Verification of Stopping Power for Proton Transport in MCNP5. American Nuclear Society Meeting, November 2003.

[8]. Chengyu S., Chin-Cheng C., Dennis M. and Maria F. C., 2018. Monte Carlo calculation of the mass stopping power of EBT3 and EBT-XD films for protons for energy ranges of $50-400 \mathrm{MeV}$. http://DOI: $10.1002 /$ pro6.55

[9]. El-Ghossain M. O., 2017. Calculations of Stopping Power, and Range of Electrons Interaction with Different Material and Human Body Parts International Journal of Scientific \& Technology Research Volume 6, Issue 01, January 2017 ISSN 2277-8616 114 Ijstr@2017 http://www.Ijstr.Org

[10]. Alfredo A. Correa, 2018. Calculating electronic stopping power in materials from first principles.
Lawrence Livermore National Laboratory LLNLJRNL-749257

[11]. [dataset] MCNP Team, 2003. MCNP Manual: Appendix G-MCNP Data Library

[12]. Shultis J. K. and Faw R. E., 2011. Introduction to the MCNP Code. LANL

[13]. [dataset] X-5 Monte Carlo Team, 2003. MCNP - A General Monte Carlo N-Particle Transport Code, Version 5 Volume I: Overview and Theory, LA-UR03-1987

[14]. Bello O. M., Wan Hassan W. M. S. and Nor N. B. M., 2019. Analytic Estimation of the Anatomical Data for Malaysian Radiation Adult Phantom. Unpublished Coference Presentation: 5th ICRP International Symposium on the System of Radiological Protection, Adelaide Convention Centre, Australia. 17 - 21 November 2019,

[15]. Eckerman, K., Harrison J., Menzel C. H. and Clement C. H. (Ed), 2012. Compendium of Dose Coefficients based on ICRP Publication 60. Publication 119 Volume 41, Supplement 1

[16]. Pedro A., Seuntjens J. P. and Ervin B. P., 2003. Chapter 9: Calibration of Photon and Electron Beams, in: Ervin B. P. (Ed) Review of Radiation Oncology Physics: A Handbook for Teachers and Students. IAEA, Austria.

\begin{tabular}{|c|c|c|c|c|c|c|c|c|c|c|c|c|c|}
\hline Energy, $\mathrm{Me}$ & 0.1 & 0.2 & 0.3 & 0.4 & 0.5 & 0.6 & 0.8 & 1 & 2 & 4 & 6 & 8 & 10 \\
\hline Lung & $6.11 \mathrm{E}-04$ & 43E-04 & $1.41 \mathrm{E}-03$ & $1.86 \mathrm{E}-03$ & $31 \mathrm{E}-03$ & $2.71 \mathrm{E}-03$ & $3.47 \mathrm{E}-03$ & $4.19 \mathrm{E}-03$ & $6.84 \mathrm{E}-03$ & $9.52 \mathrm{E}-03$ & $1.05 \mathrm{E}-02$ & $1.05 \mathrm{E}-02$ & $1.03 \mathrm{E}-02$ \\
\hline & & & & & & & & & & & & & \\
\hline & & & & & & & & & & & & & \\
\hline & & & & & & & & & & & & & \\
\hline $\mathrm{H}$ & & & & & & & & $1.93 \mathrm{E}-02$ & $3.20 \mathrm{E}-02$ & $2 \mathrm{E}-02$ & & & \\
\hline Thyroid & & 3 & & & & & & $2.10 \mathrm{E}-02$ & $3.41 \mathrm{E}-02$ & & & & \\
\hline Kidney & & $67 \mathrm{E}-$ & $47 \mathrm{E}-03$ & $7.23 \mathrm{E}-03$ & $96 \mathrm{E}-03$ & & & $1.69 \mathrm{E}-02$ & $2.85 \mathrm{E}-02$ & & & & $\mathrm{E}-02$ \\
\hline Pancreas & & & & & & & & & & & & & \\
\hline $\mathrm{S}$ & & & & 03 & & & & E-02 & & & & & -02 \\
\hline Gall Bladder & & & & & & & & & & & & & \\
\hline & & & & & & & & & & $4.22 \mathrm{E}-02$ & & $4.71 \mathrm{E}-02$ & $4.61 \mathrm{E}-02$ \\
\hline Small Intestine & $2.40 \mathrm{E}-03$ & $3.94 \mathrm{E}-03$ & $6.01 \mathrm{E}-03$ & $8.08 \mathrm{E}-03$ & $1.00 \mathrm{E}-02$ & $1.18 \mathrm{E}-02$ & $1.52 \mathrm{E}-02$ & $1.84 \mathrm{E}-02$ & $3.04 \mathrm{E}-02$ & $4.29 \mathrm{E}-02$ & $4.78 \mathrm{E}-02$ & $4.88 \mathrm{E}-02$ & $4.81 \mathrm{E}-02$ \\
\hline
\end{tabular}

Table 2:- Linear Stopping Power, $\mathrm{S}(\mathrm{MeV} / \mathrm{cm})$ of the Modified Adult Female

\begin{tabular}{|c|c|c|c|c|c|c|c|c|c|c|c|c|c|}
\hline & 0.1 & 0.2 & 0.3 & 0.4 & 0.5 & 0.6 & 0.8 & 1 & 2 & 4 & 6 & 8 & 10 \\
\hline Lung & $44 \mathrm{E}-03$ & $.77 \mathrm{E}-03$ & $5.66 \mathrm{E}-03$ & $7.45 \mathrm{E}-03$ & $.22 \mathrm{E}-03$ & $1.09 \mathrm{E}-02$ & $1.39 \mathrm{E}-02$ & $1.67 \mathrm{E}-02$ & $2.74 \mathrm{E}-02$ & $3.81 \mathrm{E}-02$ & $4.18 \mathrm{E}-02$ & $.19 \mathrm{E}-02$ & $.11 \mathrm{E}-02$ \\
\hline & & & & & & & & & & & & & \\
\hline & & & & & & & & & & & & & \\
\hline & & & & & & & & & & & & & \\
\hline & & 90E-03 & $6.00 \mathrm{E}-$ & 03 & & $1.19 \mathrm{E}$ & $1.54 \mathrm{E}$ & $1.86 \mathrm{E}-02$ & $3.07 \mathrm{E}-02$ & 4.351 & $8 \mathrm{E}-02$ & & \\
\hline $\mathrm{T}$ & $2 \mathrm{E}-03$ & 12E-03 & $6.56 \mathrm{E}-03$ & $8.75 \mathrm{E}-03$ & & $1.30 \mathrm{E}-02$ & $1.68 \mathrm{E}-02$ & $2.02 \mathrm{E}-02$ & $3.28 \mathrm{E}-02$ & $4.49 \mathrm{E}-02$ & $4.88 \mathrm{E}-02$ & & $4.90 \mathrm{E}-02$ \\
\hline & & & $26 \mathrm{E}$ - & & & & & $1.62 \mathrm{E}-02$ & $2.74 \mathrm{E}-02$ & $97 \mathrm{E}-02$ & $\mathrm{E}-02$ & & \\
\hline Pancreas & $3 \mathrm{E}-03$ & $56 \mathrm{E}-03$ & $5.24 \mathrm{E}-03$ & $97 \mathrm{E}-03$ & & $1.02 \mathrm{E}-02$ & $1.33 \mathrm{E}-02$ & $1.61 \mathrm{E}-02$ & $2.69 \mathrm{E}-02$ & $3.83 \mathrm{E}-02$ & $25 \mathrm{E}-02$ & & $4.18 \mathrm{E}-02$ \\
\hline $\mathrm{S}$ & $4 \mathrm{E}-03$ & $56 \mathrm{E}-03$ & $5.36 \mathrm{E}-03$ & & & $1.06 \mathrm{E}-02$ & & $1.66 \mathrm{E}-02$ & $2.80 \mathrm{E}-02$ & $\mathrm{E}-02$ & $4.49 \mathrm{E}-02$ & & $.54 \mathrm{E}-02$ \\
\hline Gall Bladdes & 10E-03 & $75 \mathrm{E}-03$ & $5.44 \mathrm{E}-03$ & $7.39 \mathrm{E}-03$ & & $1.08 \mathrm{E}-02$ & $1.41 \mathrm{E}-02$ & $1.69 \mathrm{E}-02$ & $2.78 \mathrm{E}-02$ & $3.89 \mathrm{E}-02$ & $4.29 \mathrm{E}-02$ & $4.37 \mathrm{E}-02$ & $4.24 \mathrm{E}-02$ \\
\hline & & & & & & & & & & & & & \\
\hline Small Intestine & $2.31 \mathrm{E}-03$ & $3.78 \mathrm{E}-03$ & $5.78 \mathrm{E}-03$ & $7.77 \mathrm{E}-03$ & $9.62 \mathrm{E}-03$ & $1.14 \mathrm{E}-02$ & $1.46 \mathrm{E}-02$ & $1.77 \mathrm{E}-02$ & $2.92 \mathrm{E}-02$ & $4.12 \mathrm{E}-02$ & $4.60 \mathrm{E}-02$ & $4.69 \mathrm{E}-02$ & $4.62 \mathrm{E}-02$ \\
\hline
\end{tabular}

Table 3:- Mass Stopping Power, $\mathrm{S} / \rho(\mathrm{MeV} g-1 \mathrm{~cm} 2)$ of the Modified Adult Female 
ISSN No:-2456-2165

\begin{tabular}{|c|c|c|c|c|c|c|c|c|c|c|c|c|c|}
\hline Energy, MeV & 0.1 & 0.2 & 0.3 & 0.4 & 0.5 & 0.6 & 0.8 & 1 & 2 & 4 & 6 & 8 & 10 \\
\hline Lung & $5.89 \mathrm{E}-04$ & $9.77 \mathrm{E}-04$ & $1.48 \mathrm{E}-03$ & $1.95 \mathrm{E}-03$ & $2.48 \mathrm{E}-03$ & $2.93 \mathrm{E}-03$ & $3.76 \mathrm{E}-03$ & $4.55 \mathrm{E}-03$ & $7.52 \mathrm{E}-03$ & $1.04 \mathrm{E}-02$ & $1.16 \mathrm{E}-02$ & $1.17 \mathrm{E}-02$ & $1.15 \mathrm{E}-02$ \\
\hline Liver & $2.49 \mathrm{E}-03$ & $3.76 \mathrm{E}-03$ & $5.65 \mathrm{E}-03$ & $7.51 \mathrm{E}-03$ & $9.35 \mathrm{E}-03$ & $1.11 \mathrm{E}-02$ & $1.44 \mathrm{E}-02$ & $1.73 \mathrm{E}-02$ & $1.43 \mathrm{E}-02$ & $4.05 \mathrm{E}-02$ & $4.51 \mathrm{E}-02$ & 4.49E-02 & $4.50 \mathrm{E}-02$ \\
\hline Stomach & $2.30 \mathrm{E}-03$ & $3.77 \mathrm{E}-03$ & $5.83 \mathrm{E}-03$ & $7.67 \mathrm{E}-03$ & $9.37 \mathrm{E}-03$ & $1.12 \mathrm{E}-02$ & $1.47 \mathrm{E}-02$ & $1.75 \mathrm{E}-02$ & $2.86 \mathrm{E}-02$ & $4.11 \mathrm{E}-02$ & $4.42 \mathrm{E}-02$ & $4.41 \mathrm{E}-02$ & 4.33E-02 \\
\hline Ovaries & 2.32E-03 & $4.22 \mathrm{E}-03$ & $6.99 \mathrm{E}-03$ & $9.69 \mathrm{E}-03$ & $1.24 \mathrm{E}-02$ & $1.42 \mathrm{E}-02$ & $1.85 \mathrm{E}-02$ & $2.27 \mathrm{E}-02$ & $3.69 \mathrm{E}-02$ & $4.88 \mathrm{E}-02$ & $5.46 \mathrm{E}-02$ & $5.78 \mathrm{E}-02$ & $5.65 \mathrm{E}-02$ \\
\hline Brain & $2.33 \mathrm{E}-03$ & $4.05 \mathrm{E}-03$ & $6.24 \mathrm{E}-03$ & $8.36 \mathrm{E}-03$ & $1.03 \mathrm{E}-02$ & $1.23 \mathrm{E}-02$ & $1.60 \mathrm{E}-02$ & $1.90 \mathrm{E}-02$ & $3.11 \mathrm{E}-02$ & $4.42 \mathrm{E}-02$ & $5.00 \mathrm{E}-02$ & $5.11 \mathrm{E}-02$ & $5.04 \mathrm{E}-02$ \\
\hline Thyroid & $2.65 \mathrm{E}-03$ & $4.41 \mathrm{E}-03$ & $6.73 \mathrm{E}-03$ & $9.27 \mathrm{E}-03$ & $1.20 \mathrm{E}-03$ & $1.24 \mathrm{E}-02$ & $1.66 \mathrm{E}-02$ & $2.11 \mathrm{E}-02$ & $3.50 \mathrm{E}-02$ & $4.80 \mathrm{E}-02$ & $5.24 \mathrm{E}-02$ & $5.09 \mathrm{E}-02$ & $5.57 \mathrm{E}-02$ \\
\hline Kidney & $2.63 \mathrm{E}-03$ & $3.50 \mathrm{E}-03$ & $5.25 \mathrm{E}-03$ & $6.89 \mathrm{E}-03$ & $8.53 \mathrm{E}-03$ & $1.03 \mathrm{E}-02$ & $1.29 \mathrm{E}-02$ & $1.60 \mathrm{E}-02$ & $2.69 \mathrm{E}-02$ & $3.87 \mathrm{E}-02$ & $4.48 \mathrm{E}-02$ & $4.68 \mathrm{E}-02$ & $4.59 \mathrm{E}-02$ \\
\hline Pancreas & $2.69 \mathrm{E}-03$ & $3.67 \mathrm{E}-03$ & $5.36 \mathrm{E}-03$ & $6.71 \mathrm{E}-03$ & $8.42 \mathrm{E}-03$ & $9.89 \mathrm{E}-03$ & $1.31 \mathrm{E}-02$ & $1.60 \mathrm{E}-02$ & $2.61 \mathrm{E}-02$ & $3.74 \mathrm{E}-02$ & 4.19E-02 & $4.22 \mathrm{E}-02$ & $4.21 \mathrm{E}-02$ \\
\hline Spleen & $2.41 \mathrm{E}-03$ & $3.84 \mathrm{E}-03$ & $5.23 \mathrm{E}-03$ & $7.28 \mathrm{E}-03$ & $8.56 \mathrm{E}-03$ & $1.07 \mathrm{E}-02$ & $1.35 \mathrm{E}-02$ & $1.60 \mathrm{E}-02$ & $2.80 \mathrm{E}-02$ & $3.92 \mathrm{E}-02$ & $4.40 \mathrm{E}-02$ & $4.52 \mathrm{E}-02$ & $4.38 \mathrm{E}-02$ \\
\hline Gall Bladder & $3.14 \mathrm{E}-03$ & $3.33 \mathrm{E}-03$ & $5.04 \mathrm{E}-03$ & $6.57 \mathrm{E}-03$ & $8.61 \mathrm{E}-03$ & $1.03 \mathrm{E}-02$ & 1.40E-02 & $1.65 \mathrm{E}-02$ & $2.70 \mathrm{E}-02$ & $3.96 \mathrm{E}-02$ & $4.35 \mathrm{E}-02$ & 4.19E-02 & 4.19E-02 \\
\hline Heart & $2.38 \mathrm{E}-03$ & $3.84 \mathrm{E}-03$ & $5.45 \mathrm{E}-03$ & 7.45E-03 & $9.39 \mathrm{E}-03$ & $1.11 \mathrm{E}-02$ & $1.45 \mathrm{E}-02$ & $1.73 \mathrm{E}-02$ & $3.86 \mathrm{E}-02$ & $4.04 \mathrm{E}-02$ & 4.44E-02 & $4.43 \mathrm{E}-02$ & $4.43 \mathrm{E}-02$ \\
\hline Small Intestine & $2.43 \mathrm{E}-03$ & $3.73 \mathrm{E}-03$ & $5.89 \mathrm{E}-03$ & $7.59 \mathrm{E}-03$ & $9.59 \mathrm{E}-03$ & $1.12 \mathrm{E}-02$ & $1.45 \mathrm{E}-02$ & $1.75 \mathrm{E}-02$ & $2.91 \mathrm{E}-02$ & $4.06 \mathrm{E}-02$ & $4.51 \mathrm{E}-02$ & 4.64E-02 & $4.56 \mathrm{E}-02$ \\
\hline
\end{tabular}

Table 4:- Linear Stopping Power, S (MeV/cm) of the Modified Adult Male

\begin{tabular}{|c|c|c|c|c|c|c|c|c|c|c|c|c|c|}
\hline Energy, MeV & 0.1 & 0.2 & 0.3 & 0.4 & 0.5 & 0.6 & 0.8 & 1 & 2 & 4 & 6 & 8 & 10 \\
\hline Lung & $2.36 \mathrm{E}-03$ & $3.91 \mathrm{E}-03$ & $5.94 \mathrm{E}-03$ & $7.79 \mathrm{E}-03$ & $9.93 \mathrm{E}-03$ & $1.17 \mathrm{E}-02$ & $1.50 \mathrm{E}-02$ & $1.82 \mathrm{E}-02$ & $3.01 \mathrm{E}-02$ & $4.16 \mathrm{E}-02$ & $4.62 \mathrm{E}-02$ & $4.69 \mathrm{E}-02$ & $4.60 \mathrm{E}-02$ \\
\hline Liver & $2.39 \mathrm{E}-03$ & $3.61 \mathrm{E}-03$ & $5.44 \mathrm{E}-03$ & $7.22 \mathrm{E}-03$ & $8.99 \mathrm{E}-03$ & $1.07 \mathrm{E}-02$ & $1.38 \mathrm{E}-02$ & $1.67 \mathrm{E}-02$ & $1.37 \mathrm{E}-02$ & $3.90 \mathrm{E}-02$ & $4.33 \mathrm{E}-02$ & $4.32 \mathrm{E}-02$ & $4.33 \mathrm{E}-02$ \\
\hline Stomach & $2.21 \mathrm{E}-03$ & $3.62 \mathrm{E}-03$ & $5.60 \mathrm{E}-03$ & $7.38 \mathrm{E}-03$ & $9.01 \mathrm{E}-03$ & $1.07 \mathrm{E}-02$ & $1.41 \mathrm{E}-02$ & $1.68 \mathrm{E}-02$ & $2.75 \mathrm{E}-02$ & $3.95 \mathrm{E}-02$ & $4.25 \mathrm{E}-02$ & $4.24 \mathrm{E}-02$ & $4.16 \mathrm{E}-02$ \\
\hline Ovaries & $2.23 \mathrm{E}-03$ & $4.06 \mathrm{E}-03$ & $6.72 \mathrm{E}-03$ & $9.32 \mathrm{E}-03$ & $1.19 \mathrm{E}-02$ & $1.37 \mathrm{E}-02$ & $1.78 \mathrm{E}-02$ & $2.19 \mathrm{E}-02$ & $3.55 \mathrm{E}-02$ & $4.69 \mathrm{E}-02$ & $5.25 \mathrm{E}-02$ & $5.56 \mathrm{E}-02$ & $5.43 \mathrm{E}-02$ \\
\hline Brain & $2.24 \mathrm{E}-03$ & $3.89 \mathrm{E}-03$ & $6.00 \mathrm{E}-03$ & $8.04 \mathrm{E}-03$ & $9.91 \mathrm{E}-03$ & $1.18 \mathrm{E}-02$ & $1.51 \mathrm{E}-02$ & $1.83 \mathrm{E}-02$ & $2.99 \mathrm{E}-02$ & $4.25 \mathrm{E}-02$ & $4.81 \mathrm{E}-02$ & $4.91 \mathrm{E}-02$ & $4.84 \mathrm{E}-02$ \\
\hline Thyroid & $2.55 \mathrm{E}-03$ & $4.24 \mathrm{E}-03$ & $6.47 \mathrm{E}-03$ & $8.92 \mathrm{E}-03$ & $1.08 \mathrm{E}-02$ & $1.20 \mathrm{E}-02$ & $1.60 \mathrm{E}-02$ & $2.03 \mathrm{E}-02$ & $3.36 \mathrm{E}-02$ & $4.61 \mathrm{E}-02$ & $5.04 \mathrm{E}-02$ & $4.89 \mathrm{E}-02$ & $5.36 \mathrm{E}-02$ \\
\hline Kidney & $2.53 \mathrm{E}-03$ & $3.37 \mathrm{E}-03$ & $5.05 \mathrm{E}-03$ & $6.62 \mathrm{E}-03$ & $8.20 \mathrm{E}-03$ & $9.86 \mathrm{E}-03$ & $1.24 \mathrm{E}-02$ & $1.54 \mathrm{E}-02$ & $2.58 \mathrm{E}-02$ & $3.72 \mathrm{E}-02$ & $4.31 \mathrm{E}-02$ & $4.50 \mathrm{E}-02$ & $4.42 \mathrm{E}-02$ \\
\hline Pancreas & $2.59 \mathrm{E}-03$ & $3.53 \mathrm{E}-03$ & $5.15 \mathrm{E}-03$ & $6.45 \mathrm{E}-03$ & $8.09 \mathrm{E}-03$ & $9.51 \mathrm{E}-03$ & $1.26 \mathrm{E}-02$ & $1.54 \mathrm{E}-02$ & $2.51 \mathrm{E}-02$ & $3.60 \mathrm{E}-02$ & $4.03 \mathrm{E}-02$ & $4.06 \mathrm{E}-02$ & $4.05 \mathrm{E}-02$ \\
\hline Spleen & $2.32 \mathrm{E}-03$ & $3.69 \mathrm{E}-03$ & $5.03 \mathrm{E}-03$ & $7.00 \mathrm{E}-03$ & $8.23 \mathrm{E}-03$ & $1.03 \mathrm{E}-02$ & $1.29 \mathrm{E}-02$ & $1.54 \mathrm{E}-02$ & $2.69 \mathrm{E}-02$ & $3.77 \mathrm{E}-02$ & $4.23 \mathrm{E}-02$ & $4.35 \mathrm{E}-02$ & $4.21 \mathrm{E}-02$ \\
\hline Gall Bladder & $3.02 \mathrm{E}-03$ & $3.20 \mathrm{E}-03$ & $4.84 \mathrm{E}-03$ & $6.31 \mathrm{E}-03$ & $8.28 \mathrm{E}-03$ & $9.90 \mathrm{E}-03$ & $1.35 \mathrm{E}-02$ & $1.58 \mathrm{E}-02$ & $2.60 \mathrm{E}-02$ & $3.81 \mathrm{E}-02$ & $4.18 \mathrm{E}-02$ & $4.03 \mathrm{E}-02$ & $4.03 \mathrm{E}-02$ \\
\hline Heart & $2.29 \mathrm{E}-03$ & $3.69 \mathrm{E}-03$ & $5.24 \mathrm{E}-03$ & $7.16 \mathrm{E}-03$ & $9.03 \mathrm{E}-03$ & $1.07 \mathrm{E}-02$ & $1.40 \mathrm{E}-02$ & $1.67 \mathrm{E}-02$ & $3.71 \mathrm{E}-02$ & $3.89 \mathrm{E}-02$ & $4.27 \mathrm{E}-02$ & $4.26 \mathrm{E}-02$ & $4.26 \mathrm{E}-02$ \\
\hline Small Intestine & $2.34 \mathrm{E}-03$ & $3.63 \mathrm{E}-03$ & $5.66 \mathrm{E}-03$ & 7.30E-03 & $9.22 \mathrm{E}-03$ & $1.08 \mathrm{E}-02$ & $1.39 \mathrm{E}-02$ & $1.68 \mathrm{E}-02$ & $2.80 \mathrm{E}-02$ & $3.90 \mathrm{E}-02$ & $4.33 \mathrm{E}-02$ & $4.46 \mathrm{E}-02$ & $4.38 \mathrm{E}-02$ \\
\hline
\end{tabular}

Table 5:- Mass Stopping Power, $\mathrm{S} / \rho\left(\mathrm{MeV} \mathrm{g}^{-1} \mathrm{~cm}^{2}\right)$ of the Modified Adult Male 